\title{
Solution to Nonlinearity in LTE RoF System for an Efficient DAS Topology: A Comprehensive Review (Invited)
}

\author{
T. Kanesan, Member, IEEE, R. Mohamad, S. M. Mitani, H. M. Hizan, W. P. Ng, Z. Ghassemlooy, \\ P. A. Haigh, S. Rajbhandari, Son T. Le, and Gee-Kung Chang, Fellow, IEEE
}

\begin{abstract}
In this paper, a review on radio-over-fiber (RoF) technology is conducted to support the exploding growth of mobile broadband. An RoF system will provide a platform for distributed antenna system (DAS) as a fronthaul of long term evolution (LTE) technology. A higher splitting ratio from a macrocell is required to support large DAS topology, hence higher optical launch power (OLP) is the right approach. However, high OLP generates undesirednonlinearities, namely the stimulated Brillouin scattering (SBS). Three different aspects of solving the $S B S$ process are covered in this paper, where the solutions ultimately provided an additional $4 \mathrm{~dB}$ link budget.
\end{abstract}

Index Terms - Long Term Evolution (LTE); Radio-over-fibre (RoF); Nonlinear Compensation

\section{INTRODUCTION}

$\mathbf{I}_{\mathrm{b}}^{\mathrm{N}}$ $\mathrm{N}$ the current telecommunication sector, the mobile broadband subsector gained significant momentum at a staggering subscriber rate of 7.3 billion in 2014 and potentially growing to 10 billion subscribers by 2020, which may result in a compound annual growth rate (CAGR) of 5.4\% between 2014 and 2020 [1]. As a result, long term evolution (LTE) technology is widely deployed by many operators across the globe, where LTE or its successor the LTE-advanced (LTE-A) offer higher speed, lower latency and increased efficiency in comparis on to the predecessors of 3G/3.5G [2]. Nevertheless, the CAGR indicates a highly significant traffic growth that requires increased network densification, where the most suitable solution would be small cell networks to support the high traffic generating hotspots without requiring additional spectrum. In a macrocell scenario, distributed antenna system (DAS) topology would be a suitable candidate for small cell deployment. The concept of a DAS topology is to create multiple fronthaul transmission and reception points that are geographically distributed, and all of these points will be connected to a centralized backhaul infrastructure (macrocell in this scenario) $[3,4]$.

The seamless platform that can support the high speed small cell networks without any additional spectrum requirement in a DAS topology would be the radio-over-fiber (RoF) technology. The DAS system with RoF platform proposed for LTE small cell networks is depicted in Fig. 1, where the LTE systemfollows the 3GPP standard for $20 \mathrm{MHz}$

T Kanesan, R. Mohamad, S. M. Mitani, and H. M. Hizan are with the Telekom Malaysia R\&D, 63000 Cyberjaya, Selangor, Malaysia (e-mail: thavamaran.kanesan@ieee.org).

W. P. Ng and Z. Ghassemlooy are with the Optical Communications Research Group, NCRLab, Northumbria University, Newcastle upon Tyne, NE1 8ST, U.K.

P. A. Haigh is with the High Performance Networks Group, University of Bristol, BS8 1TR, U.K.

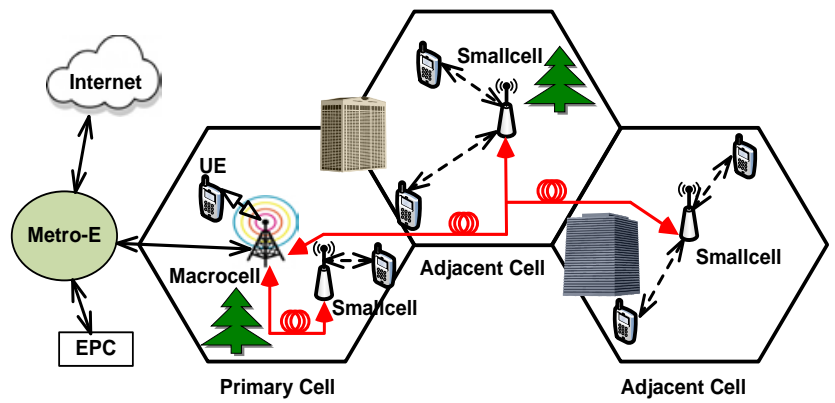

Fig. 1: LTE network with small cell fronthaul over RoF platform

bandwidth design and its relative parameters. Similar to most mobile communication scenarios, the macrocell eNodeB (eNB) is connected to the Metro-Ethernet (Metro-E) network at the backhaul layer. On the fronthaul layer, the small cells are connected to the macrocell eNB for both in-cell and outof-cell connectivity via RoF platform for a successful implementation of DAS topology. The deployment of small cells within the coverage of eNB (in-cell) is to address the cell edge issue, while the out-of-cell deployment is for densification and coverage extension purposes.

From a commercial perspective, higher number of small cells leveraging from one macrocell would provide an efficient capital expenditure (CAPEX) and operational expenditure (OPEX). Therefore, higher splitting ratio would be essential at the macrocell eNB to support many small cell networks, which in turn would require higher optical launch power (OLP) to accommodate the splitting loss and optical receiver sensitivity of the small cells network. However, OLP $>2 \mathrm{dBm}$ in a LTE RoF system introduces detrimental nonlinear distortions [5].

\section{A. Complication of Nonlinearity}

In the optical fibre propagation theory, the widely known nonlinearities are self-phase modulation (SPM), cross phase modulation (XPM), and four wave mixing (FWM), which are a composition of the Kerr effect. In the case of nonlinear scattering phenomena, there are stimulated Brillouin scattering (SBS) and stimulated Raman scattering (SRS). The focus of this paper is on single wavelength based LTE-RoF system in the C-band; therefore XPM, FWM and SRS are clearly negligible $[6,7]$. Although SPM is active in a single wavelength based system, this phenomenon will not be discuss ed further because SBS induced nonlinear distortion is

S. Rajbhandari is with the School of Computing, Electronics and Mathematics, Coventry University, Coventry, UK.

Son T. Le is with Aston Institute of Photonic Technologies, Aston University, Birmingham, B4 7ET, UK.

Gee-Kung Chang is with the School of Electrical and Computer Engineering, Georgia Inst itute of Technology, Atlanta, GA 30332 USA. 
highly prominent compared to SPM in high OLP scenario [8].

There are few methods introduced on compensating the SBS effect. Morant et al [9] introduced a spectrum management method for RoF system, which is only effective for up to $15 \mathrm{~km}$ of SMF. A co-propagating optical signal was introduced by Downie et al [8] to emulate XPM in the wavelength division multiplexing (WDM) system as a compensating agent for SBS. Apart from OLP, SBS also depends on the optical fibre effective area. Sauer et al [10] utilized an enhanced SBS threshold optical fibre, which is designed with a bigger effective area to compensate for SBS. Enhanced SBS threshold optical fibre is not applicable for LTE RoF system, because the whole idea of the proposed system is based on the existing legacy single mode fiber (SMF) backhaul to maintain a lower deployment cost [11]. In order to provide a simplified and commercially viable solution to suppress the SBS, the direct modulation based frequency dithering (DMFD) method was proposed [12].

\section{THE BASICS OF DMFD METHOD}

In this section, the basic operational principles of DMFD method will be covered, including the brief implementation process and the range of DMFD frequencies.

\section{A. DMFD Method [12]}

As a solution to the SBS distortion in LTE RoF system, a direct modulation laser is intentionally dithered or frequency chirped with a DMFD signal at certain conditional frequencies, $f_{\mathrm{d}}$ to broaden the laser linewidth. $f_{\mathrm{d}}$ only effectively dithers the laser at the condition of $\left\{f_{\mathrm{d}}<<f_{\mathrm{RF}}\right\}$ as specified earlier, where $f_{\mathrm{RF}}$ is the frequency of the LTE signal. If $\left\{f_{\mathrm{d}}>f_{\mathrm{RF}}\right\}$, then $f_{\mathrm{d}}$ will not generate the dithering phenomenon due to the existing $2^{\text {nd }}$ order harmonics in that frequency region. In other words, the laser has already experienced frequency chirping from the modulation of the LTE signal and its $2^{\text {nd }}$ order harmonics altogether. Thus, in our work, DMFD signal was generated at $f_{\mathrm{d}}$ of $100 \mathrm{MHz}$ in a sinus oidal format, which maintains the $\left\{f_{\mathrm{d}}<<f_{\mathrm{RF}}\right\}$ condition. The LTE signal is then combined with the DMFD signalprior to the process of direct modulation.

Fig. 2 (a) shows a broad frequency range where the decrease in the noise floor with DMFD method can be clearly observed for both DMFD signal and LTE signal. The harmonics and IMDs associated with the DMFD method can be easily filtered at small cell networks prior to the transmission to user equipment (UE). Additional details on the dynamic range and system linearity for LTE RoF system can be found in [11]. In order to further clarify on the SBS suppression, an enhanced version of LTE signal is shown in Fig. 3(b). The effectiveness of the DMFD method is clearly shown through the reducing out-of-band emission. The following section explores the performance improvement for a range of dithering frequency signals.

\section{B. DMFD Signal Frequency [5]}

In section II.A, the condition for $f_{\mathrm{d}}$ is determined to be $\left\{f_{\mathrm{d}}\right.$ $\left.<<f_{\mathrm{RF}}\right\}$. In order to further investigate the DMFD signal, an optimization of the dithering signal frequency and power was carried out. Fig. 3 presents the optimization of the dithering signal at OLP of $10 \mathrm{dBm}$ with a transmission span of $10 \mathrm{~km}$, and the corresponding error vector magnitude (EVM) response for the LTE signal. The aim was to achieve an EVM of $8 \%$ according to the 3GPP LTE requirement [13]. The $x$ axis of Fig. 3 is the varying frequency of the dithering signal
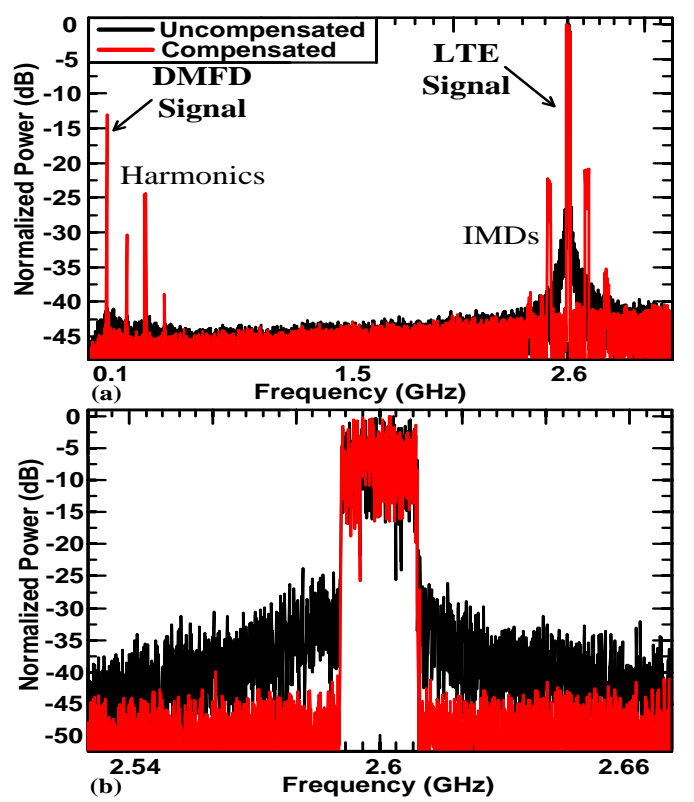

Fig. 2 [10]: Spectrum of the received signal with/without SBS compensation, (a) spectrum of LTE and DMFD signals, and (b) enhanced view of LTE signal.

with RF power in the $y$-axis, and the response of the variation is shown in z-axis as the LTE signal EVM.

In Fig. 3, launching the DMFD signal between $100 \mathrm{kHz}$ and $14 \mathrm{MHz}$ significantly increased the EVM rate. At $0 \mathrm{dBm}$ RF power and $100 \mathrm{kHz} f_{\mathrm{d}}$ increased the EVM rate to $\sim 4.98 \%$, while increasing the power to $10 \mathrm{dBm}$ resulted in EVM of $\sim 49.4 \%$. Transmitting the dithering signal at $100 \mathrm{kHz}$ does compensate the SBS, but not effectively. This is because, the intermodulation (IMD) product arising from the mixing of dithering signal at $100 \mathrm{kHz}$ and LTE signal at $2.6 \mathrm{GHz}$ falls within the $20 \mathrm{MHz}$ bandwidth of LTE signal.

It is clear that as $f_{\mathrm{d}}$ increased, the EVM decreased until the transition at $15 \mathrm{MHz}$, where the EVM completely dropped to $\sim 1.48 \%$ at $0 \mathrm{dBm} \mathrm{RF}$ power. The mixing between dithering and LTE signals at $15 \mathrm{MHz}$ and $2.6 \mathrm{GHz}$, respectively, resulted in the IMD product at $2.585 \mathrm{GHz}$, which is the explicit out-of-band IMD re-growth point. From a dithering signal frequency of $15 \mathrm{MHz}$ to $2.5 \mathrm{GHz}$, the observed EVM is as low as $\sim 1.48 \%$ at $0 \mathrm{dBm}$ of RF power, and can be further improved to $\sim 1.35 \%$ by increasing the RF power to $10 \mathrm{dBm}$.

For a dithering signal above $2.5 \mathrm{GHz}$, the effect of SBS compensation reduced as the frequency chirping had already occurred in that frequency range by modulating the LTE signal. A sharp peak can be observed when the dithering signal reached $2.6 \mathrm{GHz}$, which is due to the modulation within the bandwidth of the LTE signal. Launching the dithering signal above $2.6 \mathrm{GHz}$ resulted in an average EVM of $\sim 6.45 \%$, which achieved a close proximity with the uncompensated EVM of $\sim 6.57 \%$. The overall optimization of dithering method has shown that the frequency should not be lower and higher than $15 \mathrm{MHz}$ and $2.5 \mathrm{GHz}$, respectively, hence the expression of $\left\{f_{\mathrm{d}}<<f_{\mathrm{RF}}\right\}$ can be rewritten as $\left\{f_{\mathrm{L}}<\right.$ $\left.f_{\mathrm{d}}<f_{\mathrm{RF}}\right\}$, where $f_{\mathrm{L}}$ represents the dithering boundary limit of $14 \mathrm{MHz}$. 


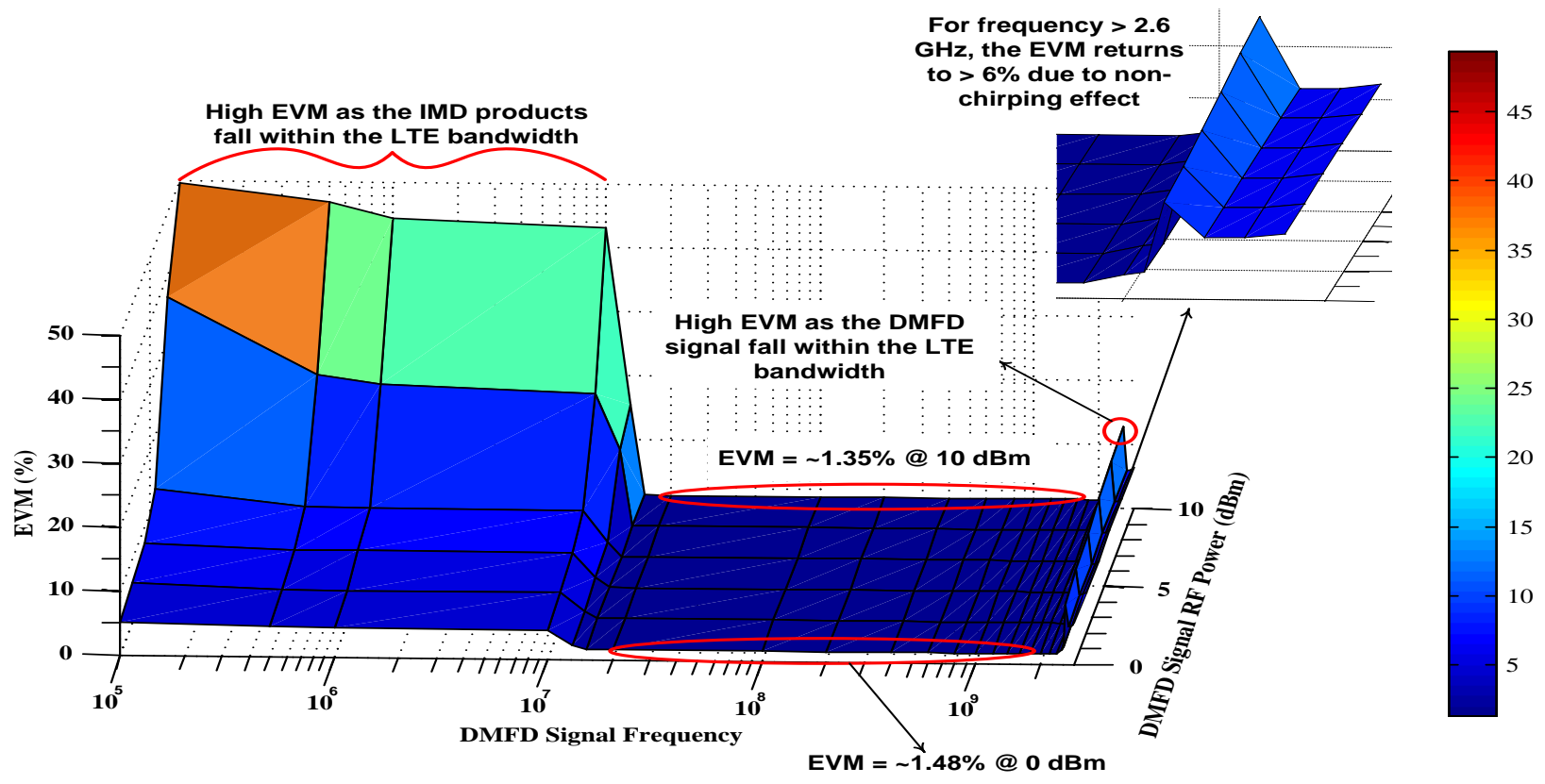

Fig. 3 [3]: Optimization of DMFD signal frequency and RF power, and its relative impact on the EVM of LTE-RoF signal

\section{DMFD Vs EMFD $[5,14]$}

Since the DMFD method relies on the modulation medium and chirping characteristics, this section will further explore on external modulation based frequency dithering (EMFD) and followed by a performance comparison with DMFD. The comparison was essential in determining if the intentional frequency chirp via dithering will further deteriorate direct modulation system over external modulation, simply because external modulation is deemed to be chirpless [15].

Figs. 4 depicts the OLP against EVM measurement for 64-QAM system modulated onto DMFD and EMFD topologies, and transmitted over 10, 35, and $50 \mathrm{~km} \mathrm{SMF}$ spans. There are three major distinctive regions, see Fig. 4, namely; (I) linear region- positive frequency chirp (PFC) and chromatic dispersion (CD) induced distortions, (II) intermixing region- reduced distortion achieved by interaction between CD and PFC with SPM and SBS, and finally (III) nonlinear region- nonlinearity based distortion from SPM and SBS effects. Regions I and II are PFC dependents. Although frequency dithering method induces frequency chirp, both DMFD and EMFD systems demonstrate resilience towards the intentional frequency chirp, thus the LTE-RoF response for regions I and II are more or less unchanged. The intentional linewidth broadening of the laser resulted in a linewidth of $\sim 37.47 \mathrm{MHz}$ [14], which indicates a smaller linewidth compared to conventional Fabry-Perot laser that exhibits a linewidth in the range of $\sim 150 \mathrm{MHz}$ [16], hence the invariable characteristic in regions I and II.

Since it is clear from Fig. 4 that both DMFD and EMFD methods are only effective for the region III.B and therefore the SBS threshold for the proposed system is $\sim 6 \mathrm{dBm}$. The region III.A does not exhibit any changes from the intentional linewidth broadening for both optical modulators due to the domination of SPM. In other word, between $\sim 2 \mathrm{dBm}$ and $\sim 6$ $\mathrm{dBm}$, the nonlinear distortion is in the form of amplitude to phase coupling, with no involvement of scattering or backreflecting power. Therefore, the discussion on Fig. 4 is focused on optical launch power levels of $8 \mathrm{dBm}$ and $10 \mathrm{dBm}$ within the region III.B. Both systemarchitectures are limited to the transmission span of $50 \mathrm{~km}$, as the proposed method
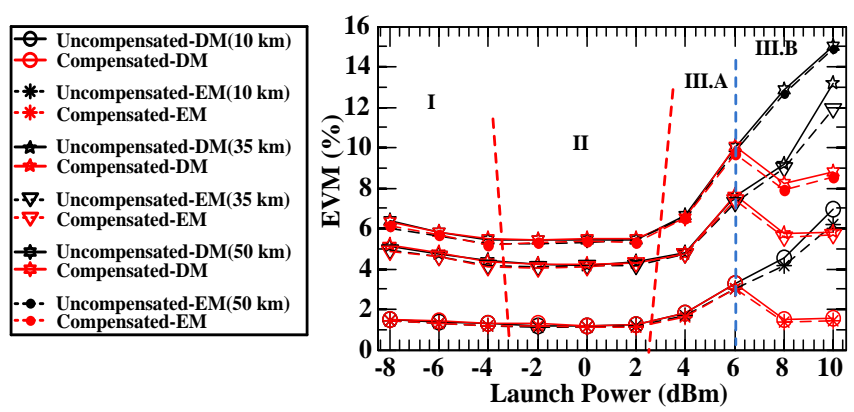

Fig. 4 [3]: OLP against EVM for SBS compensation in 64-QAM system comprising of both DMFD and EMFD methods over $10 \mathrm{~km}$ to $50 \mathrm{~km}$ transmission spans.

unable to compensate the LTE signal and achieve EVM below $8 \%$ for the transmission span of $50 \mathrm{~km}$. At the OLP of $8 \mathrm{dBm}$, EMFD topology enables the LTE RoF system to achieve EVM below $8 \%$. However, the LTE RoF systemwith the DMFD topology resulted in an EVM of $\sim 8.2 \%$, which is higher than the $8 \%$ limit. In the case of $10 \mathrm{dBm}$ OLP, both DMFD and EMFD topologies exceeded the LTE EVM limit. It is widely known that external modulation imposes higher CAPEX, therefore this study revealed that direct modulation will be a suitable candidate if the implementation allows the LTE RoF system to operate at $6 \mathrm{dBm}$ OLP with a tradeoff of $2 \mathrm{~dB}$ compared to external modulation.

\section{CONCLUSION}

In this paper, we have reviewed the nonlinear compensation of LTE-RoF system, including the DMFD method, dithering frequency optimization and the impact of employing EMFD. The advantage of applying DMFD system into an LTE RoF system is clearly demonstrated, with an optimized frequency range of $\left\{f_{\mathrm{L}}<f_{\mathrm{d}}<f_{\mathrm{RF}}\right\}$. In the review of EMFD method, the superior performance of external modulation is visible, however not significant. Ultimately, DMFD method can be employed in a brownfield deployment without major alteration to the system, and can be easily integrated into LTE RoF systemfor greenfield deployment. 


\section{REFERENCES}

[1] GSMAIntelligence. (2015, The Mobile Economy 2015. Available: http://www.gsmamobileeconomy.com/

[2] T. Nakamura, S. Nagata, A. Benjebbour, Y. Kishiyama, T. Hai, S Xiaodong, et al., "Trends in small cell enhancements in LTE advanced," Communications Magazine, IEEE, vol. 51, pp. 98-105, 2013.

[3] G. S. Gordon, M. J. Crisp, R. V. Penty, and I. H. White, "Experimental Evaluation of Layout Designs for 33 MIMO-Enabled Radio-OverFiber Distributed Antenna Systems," Vehicular Technology, IEEE Transactions on, vol. 63, pp. 643-653, 2014.

[4] R. Heath, S. Peters, Y. Wang, and J. Zhang, "A current perspective on distributed antenna systems for the downlink of cellular systems," Communications Magazine, IEEE, vol.51,pp.161-167, 2013.

[5] T. Kanesan, W. Ng, Z. Ghassemlooy, and C. Lu, "Investigation of Optical Modulators in Optimized Nonlinear Compensated LTE RoF System," Lightwave Technology, IEEE/OSA Journal of, vol. 32, pp. 1944 - 1950, 2014.

[6] F. S. Yang, M. E. Marhic, and L. G. Kazovsky, "Nonlinear crosstalk and two countermeasures in SCM-WDM optical communication systems," Lightwave Technology, Journal of, vol. 18, pp. 512-520, 2000.

[7] M. Jaworski and M. Marciniak, "Counteracting of stimulated Brillouin scattering in externally modulated light wave AM-CATV systems," in Laser and Fiber-Optical Networks Modeling, 2000. Proceedings of LFNM 2000. 2nd International Workshop on, 2000, pp. 71-73.

[8] J. D. Downie and J. Hurley, "Experimental study of SBS mitigation and transmission improvement from cross-phase modulation in 10.7 Gb/s unrepeatered systems," Opt. Express, vol. 15, pp. 9527-9534, 2007.

[9] M. Morant, T. Quinlan, S. Walker, and R. Llorente, "Complete mitigation of brillouin scattering effects in reflective passive optical net works using triple-format OFDM radio signals," in Optical Fiber Communication Conference and Exposition (OFC/NFOEC), 2011 and the National Fiber Optic Engineers Conference, 2011, pp. 1-3.

[10] M. Sauer, A. Kobyakov, and A. B. Ruffin, "Radio-Over-Fiber Transmission With Mitigated Stimulated Brillouin Scattering" Photonics Technology Letters, IEEE, vol. 19, pp. 1487-1489, 2007.

[11] W. P. Ng, T. Kanesan, Z. Ghassemlooy, and C. Lu, "Theoretical and Experimental Optimum System Design for LTE-RoF Over Varying Transmission Span and Identification of System Nonlinear Limit," Photonics Journal, IEEE, vol. 4, pp. 1560-1571, 2012.

[12] T. Kanesan, W. P. Ng, Z. Ghassemlooy, and L. Chao, "Experimental demonstration of the compensation of nonlinear propagation in a LTE RoF system with a directly modulated laser," in Communications (ICC), 2013 IEEE International Conference on, Budapest, Hungary, 2013, pp. 3884-3888.

[13] 3GPP, "EVM for LTE Repeater(3GPP TSG-RAN4 Meeting \#52, TS 36.143, Rel-8)," 2009.

[14] T. Kanesan, W. P. Ng, Z. Ghassemlooy, and C. Lu, "Impact of Optical Modulators in LTE RoF System with Nonlinear Compensator for Enhanced Power Budget," in Optical Fiber Communication Conference/National Fiber Optic Engineers Conference (OFC/NFOEC) 2013, Anaheim, California, 2013, pp. 1-3.

[15] T. Kanesan, W. P. Ng, Z. Ghassemlooy, and J. Perez, "Optimization of Optical Modulat or for LTE RoF in Nonlinear Fiber Propagation," Photonics Technology Letters, IEEE vol. 24, pp. 617-619, 2012.

[16] P. J. Probert and J. E. Carroll, "Lumped circuit model for prediction of linewidth in Fabry Perot and DFB lasers, including extemal cavity devices," Optoelectronics, IEE Proceedings J, vol. 136, pp. 22-32, 1989. 